\title{
SEROLOGIC EVIDENCE FOR AN EPIZOOTIC DENGUE VIRUS INFECTING TOQUE MACAQUES (MACACA SINICA) AT POLONNARUWA, SRI LANKA
}

\author{
ARAVINDA M. DE SILVA, WOLFGANG P. J. DITTUS, PRIYANI H. AMERASINGHE, AND FELIX P. AMERASINGHE \\ Section of Rheumatology, Department of Internal Medicine, Yale University School of Medicine, New Haven, Connecticut; \\ Department of Zoological Research, National Zoological Park, Smithsonian Institution, Washington, District of Columbia; \\ Institute of Fundamental Studies, Kandy, Sri Lanka; Department of Zoology, Faculty of Science, University of Peradeniya,
}

Peradeniya, Sri Lanka

\begin{abstract}
Dengue is one of the most rapidly emerging diseases in the tropics. Humans are the principal reservoir of dengue viruses. It is unclear if nonhuman primates also serve as a reservoir of human dengue viruses under certain conditions. In this study, a cross-sectional serologic survey was carried out to characterize the pattern of transmission of a recently identified dengue virus among toque macaques in Sri Lanka. The results indicated that an epizootic dengue virus was active among the macaques. A single epizootic had taken place between October 1986 and February 1987 during which $94 \%$ of the macaques within the $3 \mathrm{~km}^{2}$ study site were exposed to the virus. The epizootic was highly focal in nature because macaques living $5 \mathrm{~km}$ from the study population were not exposed to the virus. The transmission of dengue viruses among macaques in the wild may have important public health implications.
\end{abstract}

Dengue is the name given to four closely related but distinct arboviruses (serotypes DEN-1, DEN-2, DEN-3, and DEN-4) belonging to the genus Flavivirus in the family Flaviviridae. Over the past four decades dengue viruses have emerged as a major threat to the health of people living in many tropical areas of the world. ${ }^{1,2}$ Human dengue viruses are mostly active in urban areas where the virus is maintained through a cycle in which humans are the principal reservoir host and Aedes aegypti is the principal mosquito vector.

Human dengue infections have also been documented in rural areas in Asia, although the prevalence of infection is lower than in the urban setting. ${ }^{3-5}$ In rural areas with a low prevalence of human infection, how does the virus maintain itself? The virus may persist in the vector through direct vertical and horizontal transmission between mosquitoes. Dengue-infected female mosquitoes have genital tract infections that sometimes lead to the infection of eggs during oviposition. ${ }^{6}$ Furthermore, venereal transmission has also been noted from infected males to females. ${ }^{7}$ Another mechanism used by human arbovirus for maintaining themselves is to have additional nonhuman reservoir hosts. Yellow fever virus, a Flavivirus that shares many similarities to dengue, is maintained by an urban cycle involving Ae. aegypti and humans as well as in a sylvatic cycle with Aedes mosquitoes and nonhuman primates. ${ }^{8}$ It remains to be resolved if human dengue viruses also use nonhuman primates as reservoir hosts under certain conditions.

Dengue viruses have been isolated from monkeys and primatophilic vectors. Sylvatic circulation is common in parts of West Africa where both DEN-1 and DEN-2 viruses have been isolated from monkeys and sylvatic Aedes species. ${ }^{8}$ However, the sylvatic cycle in West Africa does not to appear to cross-over to humans because dengue infection was rare or absent among people living close to the sylvatic cycle. ${ }^{8}$ In fact, genetic analysis has shown that viruses isolated from human epidemics in Africa are distinct from the sylvatic viruses. ${ }^{9}$

Rudnick and Lim carried out studies on the ecology of dengue in Malaysia. ${ }^{10}$ These studies led to the isolation of DEN-1, DEN-2, and DEN-4 viruses from sentinel monkeys (Presbytis obscura and Macaca fascicularis). Dengue virus was also isolated from Ae. albopictus, a rural vector found at ground level, as well as from other primatophilic Aedes species found at the canopy level. Based on these findings, Rudnick has proposed that in Southeast Asia dengue viruses are maintained in a primal cycle involving monkeys and vectors of the Ae. niveus group that feed at the canopy level. ${ }^{10}$ Rudnick also proposed that the rural dengue vector (Ae. albopictus) may introduce the sylvatic virus into the human population. ${ }^{10}$ Much of the work in Malaysia was done in the 1960s and the sylvatic and human dengue viruses were not sequenced to establish the relationship between human and sylvatic dengue viruses in Asia.

Dengue is an important cause of human morbidity and mortality in Sri Lanka. ${ }^{11,12}$ While dengue viruses are primarily active in the urban areas in the southwestern part of the island, serologic surveys indicate low-level human exposure in other parts of the country. ${ }^{11}$ Four species of nonhuman primates are present in Sri Lanka, and they often live in proximity to people in rural villages. ${ }^{13}$ The sociodemography of a population of toque macaques (Macaca sinica) at Polonnaruwa has been intensely studied by Dittus and others since $1968 .^{13}$ Evidence for a sylvatic dengue cycle among these macaques was recently reported by Peiris and others, who found that $94 \%$ of the monkeys tested had antibodies that neutralized DEN-2 virus. ${ }^{14}$ The objective of the current study was to further characterize the pattern of infection of this virus among macaques at Polonnaruwa. We report here that the virus causes epizootics among the macaques, rather than being enzootic as was previously thought.

\section{MATERIALS AND METHODS}

Study site and macaque population. The population of toque macaques used in the current study inhabit the natural dry evergreen forest within the Nature and Archaeological Reserve at Polonnaruwa (Figure 1). ${ }^{13}$ The behavior, ecology, demography, and genetics of these wild monkeys have been intensively studied since 1968 by Dittus and others. ${ }^{13,15-18}$ In 1995 , the population comprised nearly 1,000 monkeys distributed among some 28 social groups. All the macaques in the population have been individually identified and the dates of birth and life histories of nearly all the animals are 


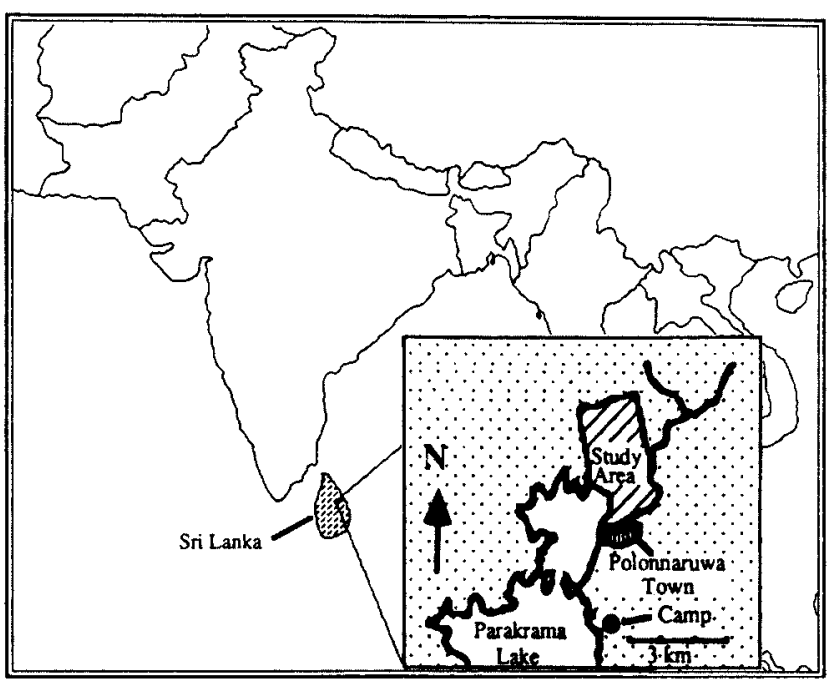

FIGURE 1. Location and map of study site (adapted from Hoelzer and others ${ }^{20}$ ). The position of the study site and camp site at Polonnaruwa are indicated.

known. The ages of animals whose births were not observed, such as immigrants into the study population, were estimated based on known relationships between morphologic development and age. ${ }^{19}$

Macaque serum samples. Blood was collected for genetic and epidemiologic analyses by trapping the macaques in cages and tranquilizing them with ketamine hydrochloride as previously described. ${ }^{20}$ Blood was collected by venipuncture and separated into serum the same day and stored in liquid nitrogen. Sera collected in 1995, 1987, and 1986 were used in the current study. Control sera samples from laboratory rhesus macaques $(M$. mullata) infected with dengue or yellow fever viruses were provided by the Yale Arbovirus Research Unit (YARU) (New Haven, CT).

Serologic tests. An ELISA was used to screen macaque sera for dengue antibody. Each plate was coated for $2 \mathrm{hr}$ at $37^{\circ} \mathrm{C}$ with the $4 \mathrm{G} 2$ anti-flavivirus monoclonal antibody (provided by YARU). ${ }^{21}$ The plate was washed and incubated with a blocking solution ( $5 \%$ horse serum in phosphate-buffered saline [PBS]) for $15 \mathrm{~min}$ at $37^{\circ} \mathrm{C}$. Next, the plates were incubated overnight at $4^{\circ} \mathrm{C}$ with the virus antigen. Flavivirus antigens (DEN-1, DEN-2, DEN-3, DEN-4, yellow fever, Japanese encephalitis, and Zika viruses) were prepared and provided by YARU. Since the antigens were prepared in mouse brain, uninfected mouse brain antigen was used as a negative control. The following day the unbound antigen was washed away with PBS containing $0.5 \%$ Tween 20 (PBS/Tween) and the macaque sera were added at a 1:100 dilution and incubated for $1 \mathrm{hr}$ at $37^{\circ} \mathrm{C}$. The plates were washed with $\mathrm{PBS} /$ Tween and incubated for $30 \mathrm{~min}$ at $37^{\circ} \mathrm{C}$ with horseradish peroxidase-conjugated goat anti-monkey immunoglobulin (Sigma Biosciences, St. Louis, MO). Finally, the plates were developed by adding commercially prepared peroxidase substrate (Zymed Laboratories, San Francisco, CA). Each serum sample was tested in duplicate against each of the test antigens. Sera that gave an optical density (OD) that was at least four times greater than the normal mouse brain negative control were designated as positive sera.

\section{RESULTS}

Enzyme-linked immunosorbent assay for detecting dengue antibody in monkeys. Flavivirus antigens are notorious for generating antibodies that cross-react between Flaviviruses. Thus, it was important to determine if the ELISA used to screen macaque sera was capable of distinguishing between Flaviviruses. For this purpose sera from laboratory monkeys infected with dengue or yellow fever were used. As expected, the sera cross-reacted with Flavivirus antigens other than the virus used in the original infection (Figure 2). However, for each of the sera the highest signal was observed in the presence of antigen from the infecting virus (Figure 2). Thus, although cross-reactivity was observed, it was possible to clearly distinguish the infecting Flavivirus from other Flaviviruses. These results validate the use of this ELISA as a means of screening macaques for Flavivirus antibodies, as well as characterizing at a serologic level the relationship of macaque Flaviviruses to known Flaviviruses.

Analysis of 1995 sera. Two hundred forty-four serum samples collected between July and October 1995 were screened for the presence of antibodies against dengue-2 virus. Twenty-one percent (52 of 244) of the animals tested positive for dengue virus antibody. The macaque sera were also tested using antigens from yellow fever, Japanese encephalitis, and Zika viruses. Most of the dengue-positive sera also cross-reacted with these antigens, although the signal was consistently less than the signal for the dengue-2 virus antigen (Figure 3). The mean ODs for the dengue virus antigen were $1.7,3$, and 3.3 times greater than the mean ODs for the yellow fever, Japanese encephalitis, and Zika virus antigens, respectively (Figure 3). None of the dengue virus-negative sera bound to the other Flavivirus antigens. These results indicate that the Flavivirus infecting the macaques was antigenically most closely related to dengue virus and was distinct from the antigenic complexes that include Japanese encephalitis and Zika viruses.

The ELISAs were also performed to determine the dengue virus serotype circulating among the macaques. Plates were coated with antigen prepared with the different dengue virus serotypes. Five representative positive sera were selected and serial dilutions of each sera was tested with the different dengue viral antigens. The positive sera cross-reacted with the four antigens and, in spite of the serial dilutions, it was not possible to single out any particular antigen as belonging to the serotype responsible for the original infection.

Analysis by sex and social group (1995 sera). The ratio of males to females in the study sample (1:1.13) was similar to the ratio of males to females among the seropositive animals $(1: 1.38)$, indicating that both sexes were equally susceptible to the virus. The 244 animals tested in this study belonged to 16 of the 28 different social groups living within the study area. The data were analyzed to determine the prevalence of infection in the different social groups. Animals from all the groups tested had at least one infected individual and the prevalence of infection in the different groups ranged from $6 \%$ to $50 \%$ (Table 1), depending on the number of group members nine years of age or older. Thus, 


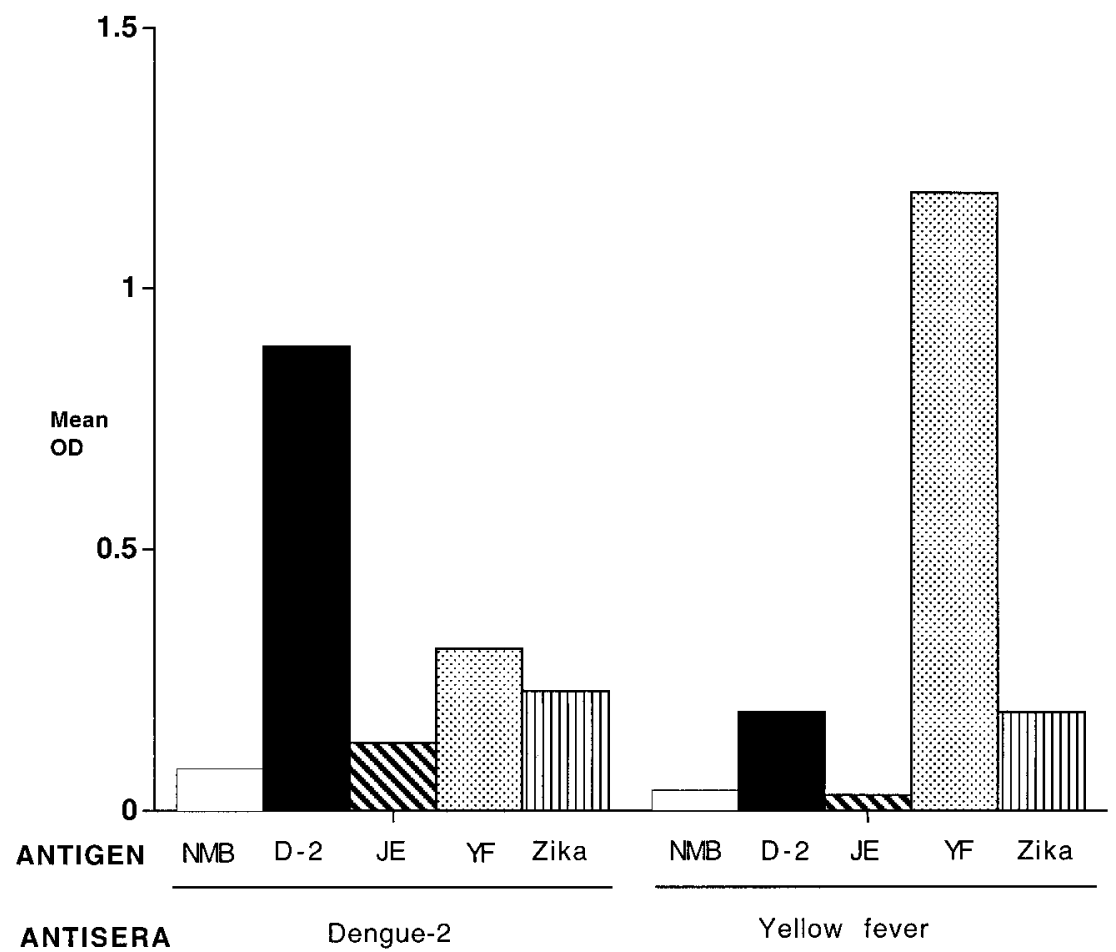

FIGURE 2. The ELISA for detecting Flavivirus antibodies in macaque sera. The ELISA plates were coated with normal mouse brain (NMB) and dengue-2 (D-2), Japanese encephalitis (JE), yellow fever (YF), and Zika virus antigens. Each plate was incubated with sera from a monkey infected with yellow fever or DEN-2 viruses. Each serum and antigen combination was tested in duplicate. OD = optical density.

the virus was active in an area encompassing the entire study site, if not a larger area.

Analysis by age (1995 sera). The age of the animals tested for dengue virus antibody ranged from less than onemonth-old infants to 33-year-old adults. The prevalence of infection in the different age groups of macaques was analyzed. Figure 4 shows the number of animals tested in each

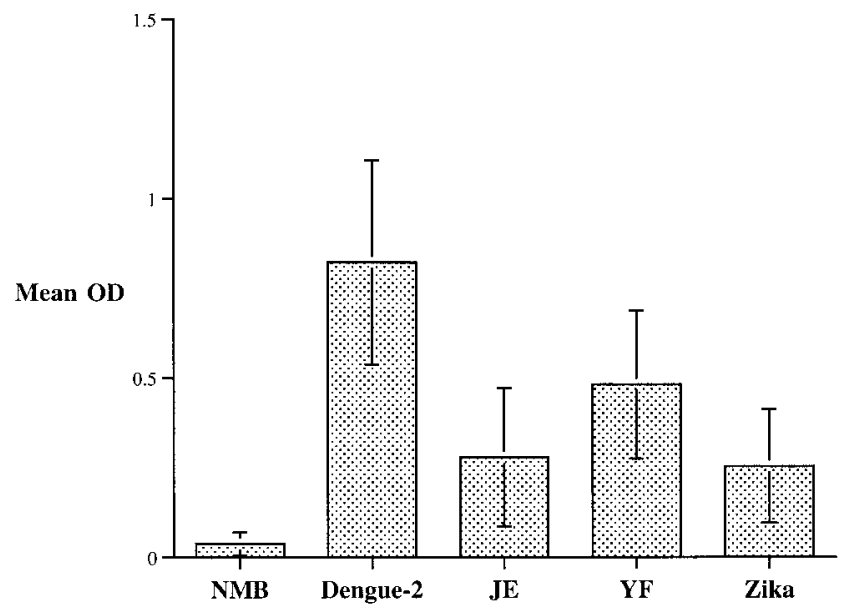

FIGURE 3. Analysis of sera showing that flavivirus antibody-positive macaque sera bound best to the dengue virus antigen. The 52 flavivirus antibody-positive macaque sera were tested against normal mouse brain (NMB), Dengue-2, Japanese encephalitis (JE), yellow fever (YF), and Zika virus antigens. The mean value obtained from all 52 positive sera against each of the antigens is displayed. Bars show the mean $\pm \mathrm{SD}$. OD $=$ optical density. age cohort, as well as the number of animals exposed to the virus. Very few $(2.6 \%)$ animals in the eight year of age and younger cohorts were exposed to the virus whereas $88 \%$ of the animals in the nine year of age and older cohorts were exposed to the virus. The pattern of exposure in the different age cohorts is highly suggestive of an epizootic virus that was active 8-9 years (between 1986 and 1987) prior to the time when the blood was collected in 1995. Thus, all the animals that were born after the epizootic in 1986-1987, which includes $80 \%$ of the animals in the eight-year-old co-

TABLE 1

Prevalence of dengue antibody among macaques from different social groups in Polonnaruwa, Sri Lanka

\begin{tabular}{lccc}
\hline Group ID & No. tested & No. positive & $(\%)$ \\
\hline 22B & 13 & 3 & $(23)$ \\
22D & 18 & 6 & $(33)$ \\
22N & 15 & 4 & $(27)$ \\
A & 17 & 1 & $(6)$ \\
B & 38 & 6 & $(16)$ \\
CH1 & 13 & 4 & $(31)$ \\
CF & 13 & 3 & $(23)$ \\
D1 & 38 & 6 & $(16)$ \\
D2 & 9 & 2 & $(22)$ \\
F1 & 14 & 4 & $(29)$ \\
F2 & 6 & 3 & $(50)$ \\
F3 & 6 & 1 & $(17)$ \\
H2 & 9 & 2 & $(22)$ \\
J & 4 & 1 & $(25)$ \\
M1 & 25 & 5 & $(20)$ \\
M2 & 6 & 1 & $(17)$ \\
Total & 244 & 52 & $(21)$ \\
\hline
\end{tabular}




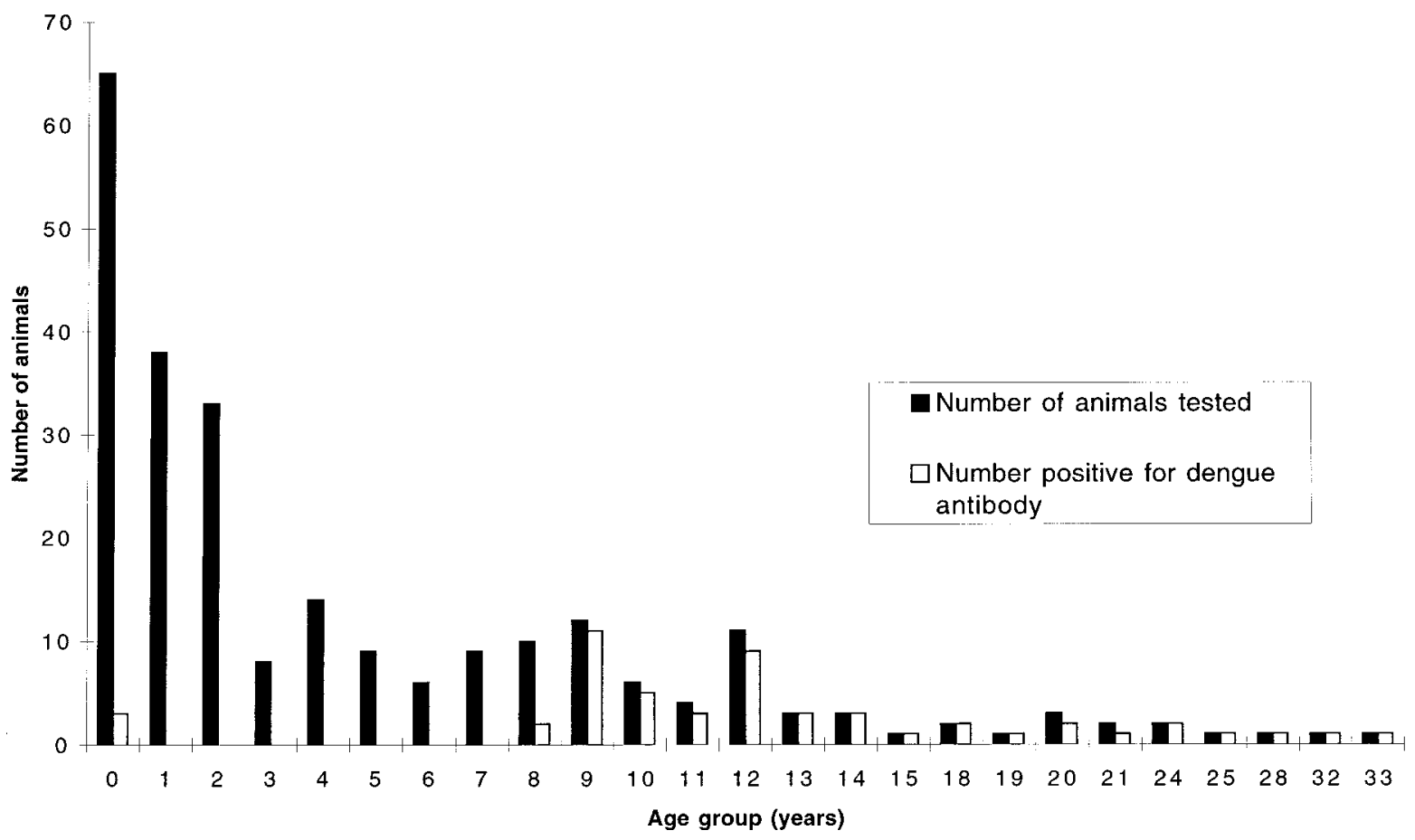

FIGURE 4. Prevalence of dengue antibody among macaques in different age groups (1995 sera).

hort and all of the animals in the seven-year-old and younger cohorts, were not exposed to the virus. The three positive animals in the 0 -year-old cohort are almost certainly due to maternal antibody. In fact, two of the mothers of these infants were tested and found to be positive animals more than nine years of age. The mother of the third positive infant was not tested in this study. Prior to the point at which transmission stopped in 1986-1987, there might have been a single epizootic that exposed almost all the individuals in the

TABLE 2

Dengue antibodies among macaques in the eight-year-old cohort (1995 sera) in Polonnaruwa, Sri Lanka

\begin{tabular}{clc}
\hline Animal ID number & Date of birth & ELISA result \\
\hline 2739 & Aug 16, 1986 & + \\
2747 & Dec 28, 1986 & + \\
2941 & Jan 5, 1987 & - \\
2801 & Jan 6, 1987 & + \\
2884 & Feb 4, 1987 & - \\
2923 & Feb 11, 1987 & - \\
2952 & Feb 21, 1987 & - \\
2789 & Feb 26, 1987 & - \\
2700 & Mar 10, 1987 & - \\
3056 & Apr 7, 1987 & - \\
2845 & Apr 8, 1987 & - \\
2930 & Apr 8, 1987 & - \\
2781 & Apr 14, 1987 & - \\
2757 & Apr 27, 1987 & - \\
2925 & May 1, 1987 & - \\
2833 & May 3, 1987 & - \\
2759 & May 9, 1987 & - \\
3094 & May 10, 1987 & - \\
2920 & May 19, 1987 & - \\
3095 & May 31, 1987 & - \\
\hline
\end{tabular}

population or the virus may have been enzootic among the macaques.

Detailed analysis of eight-year-old animals (1995 sera). To determine more accurately when this virus stopped circulating among the macaques, all the eight-year-old animals bled in 1995 (this sample included 10 animals from the original 246 animal samples, as well as 10 animals not included in the original sample, but also trapped in 1995) were tested for dengue- 2 virus antibody (Table 2). Three of the 20 animals in the eight-year-old cohort were positive for dengue virus antibody. The dates of birth of the eight-year-old animals and their serologic status is presented in Table 2. It is clear from these data that only the oldest animals in this cohort (i.e., those born before January 6, 1987) were exposed to this virus, while none of the animals born after February 4, 1987 were exposed. These results suggest that transmission abruptly ceased among the macaques starting sometime between January and February 1987.

Analysis of 1986 and 1987 sera. The serologic analysis of macaque samples collected in 1995 indicate that the animals were exposed to a dengue virus whose transmission abruptly ceased before February 1987. Prior to February 1987, the virus may have been responsible for a single large epizootic that occurred towards the end of 1986 or beginning of 1987 or the virus may have been enzootic among the macaques. To further characterize the pattern of transmission during the critical period between 1986 and 1987, blood samples collected in September 1986 (pre-epizootic?) and blood samples collected between June and August 1987 (post-epizootic) were tested for dengue virus antibody. These results, which are presented in Table 3, confirm that $81 \%$ of the macaques were infected due to a single epizootic that 
TABLE 3

Prevalence of dengue antibody among macaques bled in 1986 and 1987 in Polonnaruwa, Sri Lanka

\begin{tabular}{ccc}
\hline & \multicolumn{2}{c}{ Date bled } \\
\cline { 2 - 3 } & 1986 (September) & 1987 (July-September) \\
\hline No. of animals tested & 16 & 44 \\
No. positive (ELISA) & $2 *(12 \%)$ & $41(93 \%)$ \\
\hline$*$ The two positive animals were 15 and 20 years old at the time of bleeding in 1986.
\end{tabular}

took place at the end of 1986 . Of the 16 animals sampled in September 1986, only two older animals were seropositive. In contrast, when the same population was sampled in 1987 the seroprevalence was $94 \%$ (41 of 44), indicating that a major epizootic took place between 1986 and 1987. The two positive animals in the 1986 sample indicate that sometime in the past there may have been another epizootic, a possibility that is supported by the observation that the two positive animals (15 and 20 years of age) were among the oldest of the animals in the 1986 sample.

Timing of infection in relation to weather. Most rainfall at Polonnaruwa normally occurs between October and January due to inter-monsoonal convectional rains in October and the northeast monsoon from November to January (Table 4). February is normally dry with clear skies. The onset of the virus infection among the macaques at Polonnaruwa after September 1986 and its cessation before February 1987 coincided with the rainy season. The fact that transmission took place during the rainy season is consistent with the virus being transmitted by a mosquito vector.

Analysis of camp animals. The tests performed with sera from macaques living within the study site at Polonnaruwa indicated that almost all the animals were exposed to a dengue virus towards the end of 1986. Was this a focal event restricted to animals at the study site, or did the 1986 epizootic represent an island-wide incident? To examine the geographic extent of the epizootic, toque macaque sera collected in 1986 and in 1995 from animals at a site $5 \mathrm{~km}$ from the study site and separated from the study site by cultivation (Figure 1, camp site) were tested for dengue virus antibody. These animals have not been extensively studied and biographic data including the dates of birth were not available. The ages of these animals was estimated using morphologic criteria as previously described. ${ }^{19}$ Two seropositive older animals and two seronegative younger animals from the study site were also tested in this experiment as controls. None of the animals trapped at the camp site in 1986 were positive. More importantly, none of the animals trapped in 1995, many of whom were estimated to be more than nine years of age, were positive for dengue virus antibody. This result was in sharp contrast to the data obtained for animals at the study site where nearly all the older animals were positive. These data indicate that the 1986-1987 epizootic was focal in nature and restricted to animals living in the immediate proximity of the Polonnaruwa study site.

\section{DISCUSSION}

This study has provided serologic evidence for an epizootic dengue virus among toque macaques in Sri Lanka. One
TABLE 4

Rainfall at Polonnaruwa, Sri Lanka before, during, and after the northeast monsoon of 1986-1987

\begin{tabular}{ccc}
\hline Month & Rainfall $(\mathrm{mm})$ & No. of days of rain \\
\hline 1986: August & 21 & 3 \\
September & 6 & 1 \\
October & 276 & 10 \\
November & 125 & 8 \\
December & 350 & 18 \\
1987: January & 101 & 9 \\
February & 18 & 1 \\
March & 61 & 3 \\
\hline
\end{tabular}

must be cautious when using serologic data to identify Flaviviruses because antibodies in response to Flavivirus infections are notorious for cross-reacting with other Flaviviruses. However, the available data suggest that the macaque virus is a dengue virus. First, while four Flavivirus antigens (dengue, Japanese encephalitis, Zika, and yellow fever viruses) were tested in the current study, the highest signal was observed consistently with the dengue antigen. Second, and more importantly, in the previous study by Pieris and others, they performed neutralization tests and observed that all the positive sera neutralized dengue- 2 virus (64 of 64), while very few sera neutralized JE (3 of 64) or West Nile ( 1 of 64) viruses. ${ }^{14}$ Based on these results, it is reasonable to designate the macaque virus as a dengue virus. The unambiguous identification of the agent is only possible once the virus has been isolated from macaques. Unfortunately, attempts to isolate the virus from macaque sera have so far proven unsuccessful. As in humans, dengue is an acute infection in monkeys and the virus does not persist in primates. $^{22}$ The failure to isolate virus from the sera is not surprising since none of the samples were collected during the actual epizootic between October 1986 and February 1987.

In the previous study by Pieris and others, a smaller sample of the same population of macaques was tested in $1987 .{ }^{14}$ These investigators concluded that the macaques were gradually exposed to the virus over time and that by six years of age more than $90 \%$ of the animals had seroconverted. These results were consistent with a highly active, enzootic virus. The present study with blood samples collected from the same population eight years later (1995), as well as blood samples collected in 1986 and 1987, has revealed that this is an epizootic virus characterized by outbreaks infecting almost the entire population of macaques within at least a 3 $\mathrm{km}^{2}$ area followed by prolonged (at least eight years for the Polonnaruwa population) periods with no transmission. The high seroprevalence observed by Pieris and others was due to the fact that the population was sampled in 1987 immediately after a major epizootic.

Although the virus was highly infectious to macaques, the animals did not appear to have serious illness. These animals have been intensively studied and no increased mortality or obvious signs of morbidity were observed by field assistants during the period of the epizootic (Dittus WPJ, unpublished data). These findings are consistent with the agent being a dengue virus since previous field and laboratory studies have documented that infected monkeys are mainly asymptomatic 
although they do develop viremia and can serve as a reservoir host. ${ }^{8,22,23}$

The current study has raised the following important questions. What is the origin of this virus and how is the agent maintained in Sri Lanka? Over a large part of the Amazon, yellow fever virus moves in waves through monkey populations leading to epizootics in particular regions with a periodicity of 8-10 years. ${ }^{8}$ In Senegal, epizootics of dengue among monkeys were observed in 1974 and again in $1981 .^{24}$ The toque macaque is found in many forested areas of the island, excluding the climatic extremes of arid and cold. Unlike the rhesus macaque ( $M$. mulatta) of India, however, it does not cohabit with people in towns. It is conceivable that this virus may sweep through the island infecting a troop of macaques and then moving on to the next troop as immunity builds up in the original group. A troop that has been infected may again become susceptible to an epizootic as the number of naive individuals increase in the population. The population at the study site at Polonnaruwa may be poised for another epizootic since the majority (79\%) of the animals tested in 1995 did not show evidence of exposure to this virus.

When considering the reservoirs of the macaque virus, it is important to consider other vertebrates, especially primates living on the island. In addition to toque macaques, two other species of monkeys (Semnopithecus entellus and Trachypithecus vetellus) and one species of prosimian, the slender loris (Loris tardigradus), are found at the study site. In areas of sympatry, the langurs are more common than the macaques. ${ }^{25}$ The virus may be enzootic among one of these other primates and the toque macaques may simply serve as an amplifying host. However, if the virus is enzootic in another species at the study site, it is difficult to understand why there was no evidence of infection among any of the susceptible macaques over the eight-year period between 1987 and 1995. When considering the origin of the macaque virus, it is important not to forget humans, the most abundant primate on the island and a known reservoir of dengue viruses. It is possible that the epizootic in 1986 was due to cross-over of a human dengue virus to the macaque population. Future efforts will be directed towards isolating the virus from macaques for unambiguous identification and to determine the relationship of the macaque virus to dengue viruses infecting humans in Sri Lanka.

Acknowledgments: During this study we received support from colleagues in Sri Lanka and the United States. Foremost, we thank Dr. Rebeca Rico-Hesse for advice and support throughout the project. We thank Shirley Tirrell-Peck of the Yale Arbovirus Research Unit for guidance at various stages of the project. In Sri Lanka, we thank Taya Dias, Wanaja Dittus, Sunil Goonatilake, Gayan Gunewardane, Vajira Hettige, Don Melnick, Beatrice Sweeney-Perez, Anna Pethiyagoda, S. P. Ranasinghe, and Jennifer Wilcox for technical assistance. Aravinda de Silva thanks Amy Weil for encouragement.

Financial support: Field research was supported by grants to Wolfgang P. J. Dittus from the National Science Foundation (BNS9104649, 9510894), the Harry and Frank Guggenheim Foundation, Friends of the National Zoo, and Earthwatch. The laboratory studies were supported by U.S. Army grant DAMD17-94-J-4004.

Authors' addresses: Aravinda M. de Silva, Department of Microbiology and Immunology, University of North Carolina, Campus Box 7290, Chapel Hill, NC 27599. Wolfgang P. J. Dittus, Institute of Fundamental Studies, Hantana Road, Kandy, Sri Lanka. Priyani H.
Amerasinghe and Felix P. Amerasinghe, Department of Zoology, Faculty of Science, University of Peradeniya, Peradeniya, Sri Lanka. Reprint requests: Aravinda M. de Silva, Department of Microbiology and Immunology, University of North Carolina, Campus Box 7290, Chapel Hill, NC 27599.

\section{REFERENCES}

1. Monath TP, 1994. Dengue: the risk to developed and developing countries. Proc Natl Acad Sci USA 91: 2395-2400.

2. Halstead SB, 1992. The XXth century dengue pandemic: need for surveillance and research. World Health Stat Q 45: 292 298.

3. Silarug N, Foy HM, Kupradinon S, Rojanasuphot S, Nisalak A, Pongsuwant Y, 1990. Epidemic of fever of unknown origin in rural Thailand caused by influenza $A(H 1 N 1)$ and dengue fever. Southeast Asian J Trop Med Public Health 21: 61-67.

4. Rudnick A, 1965. Studies on the ecology of dengue in Malaysia: a preliminary report. J Med Entomol 2: 203-208.

5. Mehendale SM, Risbud AR, Rao JA, Banerjee K, 1991. Outbreak of dengue fever in rural areas of Parbhani district of Maharastra (India). Indian J Med Res: 93: 6-11.

6. Rosen L, Shroyer DA, Tesh RB, Freier JE, Lien JC, 1983.Transovarial transmission of dengue viruses by mosquitoes: Aedes albopictus and Aedes aegypti. Am J Trop Med Hyg 32: 11081119.

7. Rosen L, 1987. Sexual transmission of dengue viruses by Aedes albopictus. Am J Trop Med Hyg 37: 398-402.

8. Rodhain F, 1991. The role of monkeys in the biology of dengue and yellow fever. Comp Immunol Microbiol Infect Dis 14: 919.

9. Rico-Hesse R, 1990. Molecular evolution and distribution of dengue viruses type 1 and 2 in nature. Virology 174: 479493.

10. Rudnick A, Lim TW, 1986. Dengue fever studies in Malaysia. Inst Med Res Malaysia Bull 23: 127-147.

11. Vitarana, T. 1982. Viral diseases in Sri Lanka: a national overview. Mackenzie JS, ed. Viral Diseases in South East Asia and the Western Pacific. London: Academic Press, 198-204.

12. Vitarana T, 1990. Dengue haemorrhagic fever. Ceylon Med J 35: 83-87.

13. Dittus WPJ, 1977. The socioecological basis for conservation of the Toque monkey (Macaca sinica) of Sri Lanka. Prince Rainier III of Monaco, Bourne GH, eds. Primate Conservation. New York: Academic Press, 237-265.

14. Peiris JS, Dittus WPJ, Ratnayake CB, 1993. Seroepidemiology of dengue and other arboviruses in a natural population of Toque macaques (Macaca sinica) at Polonnaruwa, Sri Lanka. J Med Primatol 22: 240-245.

15. Dittus WPJ, 1979. The evolution of behaviors regulating density and age-specific sex ratios in a primate population. Behaviour 69: 265-302.

16. Dittus WPJ, 1988. Group fission among wild toque macaques as a consequence of female resource competition and environmental stress. Anim Behaviour 36: 1626-1645.

17. Cheverud JM, Dittus WPJ, 1992. Primate population studies at Polonnaruwa. II. Heritability of body measurements in a natural population of toque macaques (Macaca sinica). Am J Primatol 27: 145-156.

18. Keane B, Dittus WPJ, Melnick DJ, 1997. Paternity assessment in wild groups of toque macaques (Macaca sinica) at Polonnaruwa, Sri Lanka using molecular markers. Mol Ecol 6: 267282.

19. Dittus WPJ, Thorington Jr RW, 1981. Techniques for aging and sexing primates. Techniques for the Study of Primate Population Ecology. Washington, DC: Subcommittee on Conservation of Natural Primate Populations, National Academy of Sciences Press, 81-134.

20. Hoelzer GA, Dittus WPJ, Ashley MV, Melnick DJ, 1994. The local distribution of highly divergent mitochondrial DNA haplotypes in Toque macaques Macaca sinica at Polonnaruwa, Sri Lanka. Mol Ecol 3: 451-458.

21. Sithiprasasna R, Strickman D, Innis BL, Linthicum KJ, 1994. ELISA for detecting dengue and Japanese encephalitis viral antigen in mosquitoes. Ann Trop Med Parasitol 88: 397-404. 
22. Halstead SB, Shotwell H, Casals J, 1973. Studies on the pathogenesis of dengue infection in monkeys. 1. Clinical laboratory responses to primary infection. J Infect Dis 128: 7-14.

23. Halstead SB, Shotwell H, Casals J, 1973. Studies on the pathogenesis of dengue infection in monkeys. II. Clinical laboratory responses to heterologous infection. J Infect Dis 128: 1522.
24. Saluzzo JF, Cornet M, Adam C, Eyraud M, Digoutte JP, 1986. Dengue 2 in eastern Senegal: serologic survey in simian and human populations. 1974-1985. Bull Soc Pathol Exot Filial 79: 313-322.

25. Eisenberg JF, Muckenhirn NA, Rudran R, 1972. The relation between ecology and social structure in primates. Science 176: $863-874$ 